\title{
On the Blast Mitigation Ability of Multiple V-Shape Deflectors
}

\author{
Sebastian Stanisławek (D) and Andrzej Morka \\ Faculty of Mechanical Engineering, Military University of Technology, Warsaw 00-908, Poland \\ Correspondence should be addressed to Sebastian Stanisławek; sebastian.stanislawek@wat.edu.pl
}

Received 23 December 2019; Accepted 3 June 2020; Published 17 June 2020

Academic Editor: Yuri S. Karinski

Copyright ( $\odot 2020$ Sebastian Stanisławek and Andrzej Morka. This is an open access article distributed under the Creative Commons Attribution License, which permits unrestricted use, distribution, and reproduction in any medium, provided the original work is properly cited.

\begin{abstract}
This paper presents a 2D numerical study of v-shape deflectors subjected to high explosive charge detonation. The literature provides many papers concerning the appropriate geometry of blast protection deflectors. Such structures require a relatively high distance between the ground and the vehicle chassis. In most cases, the placement of a deflector is not possible or it cannot cover the whole area under a chassis. An interesting question that arises is to what extent a set of small deflectors would be able to mitigate blast effects. The content of this paper constitutes an answer to this question. Three analyses were conducted: (1) multiplication of triangle components, (2) effect of deflector size, and (3) geometry-induced shock dynamics. The problem was solved with the use of modelling and simulation methods, in particular, CFD-FEM implemented in the LS-DYNA code. It was considered a plain issue in computational fluid dynamics, where space discretization for each option was built with two-dimensional elements to ensure efficient calculations. Deflectors were described using a rigid wall boundary condition, and an adequate simplified detonation model was assumed. The primary measure of the results was reaction force history, with momentum transfer and pressure distribution maps considered supplementary. The studies performed showed that both minimizing the v-shape deflector size and surrounding it with adjacent structures had a negative impact on its blast mitigation effectiveness. However, for each multi-v-shape deflector, some improvement was present, and, therefore, in situations where the installation of a typical protector is not possible due to dimensional requirements, it may offer a compromise solution.
\end{abstract}

\section{Introduction}

The common use of improvised explosive devices (IEDs) and landmines in conflict zones highlights the need for better explosion-resistant vehicles. Even if an army dominates technologically, it can suffer relatively high losses due to various types of explosives [1]. The tactics used by guerrilla groups, focusing on random attacks, make vehicles' antiblast resistance a primary goal. However, achieving a suitable speed for a vehicle and the ability to move through a muddy terrain is difficult when an appropriate level of mine and fragment resistance is also required [2]. In order to reconcile manoeuvrability and protection, further development of light armoured vehicles (LAV) is desirable.

In the most common scenario, an explosion takes place under a vehicle, making its chassis responsible for the transfer of blast energy to the hull, and consequently, to the crew members. Various aspects of blast mitigation problems can be found in $[3,4]$, where, for example, the authors of [5-8] focused on an optimal material design, while the authors in the following [9-11] studied the selection and geometric features of the panels. An advanced theoretical and experimental study of the oblique shock wave reflection phenomena [12] proved the potential advantages of geometrical barriers located on the propagation route of the blast wave in terms of momentum and energy transfer. Therefore, deflectors using the shape effect are used to mitigate blast effects, with the $\mathrm{v}$-shaped hull ("V") being the most typical. Quantitative evaluation of a "V" deflector, in comparison with a flat panel, has been the subject of many papers [13-15]. The action of the protector aims to preserve a vehicle's trajectory and has been undisputedly shown to reduce injury to occupants. Some works have investigated the hull geometry [16] in order to determine the effect of deflection for different " $V$ " angles. Generally, sharp angle deflectors disperse more energy but, however, require more space under the chassis. In other 
research studies, a u-shaped hull (" $U$ ") has been considered [17-19], with deflection patterns usually compared to "V" panels. Overall, the results show that, in general, "V" plates better disperse blast waves for any type of loading and, therefore, can be successfully applied in LAVs.

The conclusions drawn from these scientific investigations resulted in the manufacturing of vehicles with improved crew survivability. One of the most significant drawbacks in deflector application is the location of the centre of gravity, which introduces new hazards for soldiers. According to [20], rollovers were the deadliest and costliest MRAP accident types, where the specific hull geometry must have been a contributory factor. Moreover, because the installation of "V" deflectors requires relatively high ground clearance, it often cannot be applied to vehicles which were not designed for this purpose. Another interesting question which arises is how the deflection phenomenon may be used when the size of the protective structure is reduced. Unfortunately, few papers $[21,22]$ deal with the problem of how a deflector-which includes a set of small objects, especially the "V" type-is able to mitigate blast effects. The following content of this paper constitutes an answer to this question.

\section{Investigation Plan}

The mitigation effect depends on many factors, including the exact position of the explosive and the particular design of a protector. A right angle $\left(90^{\circ}\right) \mathrm{v}$-shaped plate was chosen because it was considered to be a good example of an effective blast protective structure. In order to eliminate the influence of deflector material properties, a rigid body model was utilized in all analysis. The deflector width term was defined in the reference case as a v-shape span equal to $800 \mathrm{~mm}$, which is the maximum possible value which satisfies the ground clearance requirements for a typical LAV. The charge mass was assumed to be $1.5 \mathrm{~kg}$ of TNT, which provided a proper relationship between the pressure gradient behind the shock wave front and the characteristic dimension of the deflectors studied, which guaranteed sufficient differentiation of results.

In this paper, three groups of analysis results are presented. In the first group (Section 4.1), a single deflector is compared with multiple $\mathrm{v}$-shape structures where the HE charge standoff distance is measured from the deflector base. This corresponds to the most realistic situation where a ballistic panel is mounted on a vehicle chassis and a charge is buried in the ground. A variety of factors, such as charge mass and type, standoff distance, deflector material, size and geometry, vehicle mass and stiffness, and ground properties, influence the result of blast loading. However, two specific phenomena were selected for consideration in this paper, namely, flow around the $\mathrm{v}$-shape, which is dependent on neighbouring structures' presence, and oblique reflection, which may be influenced by deflector size. In order to distinguish which effect was more significant, separate research was conducted. In Section 4.2, the influence of deflector size is considered, and in Section 4.3, disturbance of air flow is studied. All the tested variants are described in Table 1, including denotations and schematic configurations.
TABLE 1: Structure configurations studied.

Symbol Deflector description (width $(\mathrm{mm})$ ) $\quad$ Scheme

Vf800 Flat (refer.)/800 (Sections 4.1, 4.2, and 4.3)

Vs800 Single/800 (Sections 4.1-4.3)

Vd800 Double/800 (Section 4.1)

$\longrightarrow$

Vt800 Triple/800 (Section 4.1)

Vq800 Quadruple/800 (Section 4.1)

\begin{tabular}{ll}
\hline Vs560 & Single/560 (Section 4.2) \\
Vs320 & Single/320 (Section 4.2) \\
Vs80 & Single/80 (Section 4.2) \\
\hline Vf800c & Single/560 - close (Section 4.3) - close (Section 4.3) \\
\hline Vs560c & Single/200 (Section 4.3) \\
\hline Vs3200 & Single/320 - close (Section 4.3) \\
\hline Vs80c & \\
\hline & \\
\hline &
\end{tabular}

The problem was solved by the application of a computational fluid dynamic method. In order to provide greater efficiency and accuracy of simulations, it was modelled in a 2D domain, which implies that transverse dimensions are treated as infinite. Though it does not fully correspond to real conditions, mutual quantitative relations between results have been conserved for the cases investigated. 
Reaction force and momentum transfer on the deflector surface were recorded and are considered the primary measures of the results. Additionally, pressure maps were used for identification of the convergent flow zones.

\section{Numerical Model Description}

The problem formulated above had to be transformed into the mathematical language, following numerical model development. The fundamental laws of mass, momentum, and energy conservation in terms of continuum mechanics can be expressed by the system of nonlinear PDEs. According to the Euler approach and neglecting any external force fields, they take the following conservative form:

$$
\begin{array}{r}
\frac{\partial \rho}{\partial t}+\nabla \cdot(\rho \mathbf{v})=0 \\
\frac{\partial(\rho \mathbf{v})}{\partial t}+\nabla \cdot(\rho \mathbf{v} \otimes \mathbf{v}-\boldsymbol{\sigma})=0 \\
\frac{\partial(\rho E)}{\partial t}+\nabla \cdot(\rho E \mathbf{v}-\boldsymbol{\sigma} \cdot \mathbf{v})=0
\end{array}
$$

where $\rho$ denotes the mass density field and $\mathbf{v}$ denotes the fluid velocity field. The total specific energy is defined as the sum of kinetic and internal energies according to

$$
E:=\frac{v^{2}}{2}+e
$$

The system of equations (1)-(3) is complemented by the constitutive relation expressing the Cauchy stress tensor, $\boldsymbol{\sigma}$, through other problem's variables. Finally, the mathematical model formulation is completed by adequate initial and boundary conditions (IBC).

The arbitrary Lagrangian-Eulerian (ALE) algorithm implemented in the LS-DYNA environment, a widely used tool for blast modelling $[13,18,21,23]$, was chosen to provide the numerical solution to the problem. The problem equations could then be rewritten in a more suitable form, where the convective and advective terms were explicitly presented, as well as taking into account the plain formulation:

$$
\begin{aligned}
\frac{\mathrm{d} \rho}{\mathrm{d} t}+\rho\left(\frac{\partial v_{x}}{\partial x}+\frac{\partial v_{y}}{\partial y}\right) & =0 \\
\frac{\mathrm{d} \rho}{\mathrm{d} t} & :=\frac{\partial \rho}{\partial t}+\left(v_{x} \frac{\partial \rho}{\partial x}+v_{y} \frac{\partial \rho}{\partial y}\right), \\
\rho \frac{\mathrm{d} v_{x}}{\mathrm{~d} t}+\frac{\partial p}{\partial x} & =0, \\
\frac{\mathrm{d} v_{x}}{\mathrm{~d} t} & :=\frac{\partial v_{x}}{\partial t}+\left(v_{x} \frac{\partial v_{x}}{\partial x}+v_{y} \frac{\partial v_{x}}{\partial y}\right), \\
\frac{\mathrm{d} v_{y}}{\mathrm{~d} t}+\frac{\partial p}{\partial y} & =0, \\
\frac{\mathrm{d} v_{y}}{\mathrm{~d} t} & :=\frac{\partial v_{y}}{\partial t}+\left(v_{x} \frac{\partial v_{y}}{\partial x}+v_{y} \frac{\partial v_{y}}{\partial y}\right),
\end{aligned}
$$

$$
\begin{aligned}
\rho \frac{\mathrm{d} e}{\mathrm{~d} t}+p\left(\frac{\partial v_{x}}{\partial x}+\frac{\partial v_{y}}{\partial y}\right) & =0, \\
\frac{\mathrm{d} e}{\mathrm{~d} t} & :=\frac{\partial e}{\partial t}+\left(v_{x} \frac{\partial e}{\partial x}+v_{y} \frac{\partial e}{\partial y}\right),
\end{aligned}
$$

and in the case of the perfect fluid model, the stress tensor simplifies to the isotropic part depending only on the pressure, which can be described by the polytrophic equation of state (EOS) in the following form:

$$
p=\rho e(\gamma-1) \text {, }
$$

where $\gamma$ denotes the ratio of specific heats. The ALE-based method splits the solution procedure into two steps: the Lagrangian time step (FEM), followed by the advection step [24]. In the first step, a solution of (5)-(8) is found which neglects the advection terms (see the right column of equations: terms in brackets). The advection algorithms are then used to calculate the transported quantities between the deformed (Lagrangian) elements and the assumed (including initial) mesh. The element-centred quantities (density, internal energy, and stress tensor) are calculated with the van Leer MUSCL (Monotone Upwind Scheme for Conservation Laws) algorithm, while the node-centred (momentum/velocity) uses the HIS (half index shift) algorithm. Assuming that the advection step is performed after every Lagrangian step, it is completely equivalent to Eulerian problem treatment.

The solution for the FEM step is constructed starting from the weak formulation (Galerkin approach) of the equations of motion, which leads to the integral form of the problem equations. The semidiscrete form of the variational equations is then obtained by application of the assumed approximation formulas with the use of base/shape functions in discrete elements for the dependent field variables (trial and test functions). The system of semidiscrete ODEs can be transformed to the final discrete (matrix) form by the application of the central difference scheme for time integration, with the time step derived from the CFL (Courant-Friedrichs-Lewy) stability condition. Furthermore, during the Lagrangian step, the mass conservation law is automatically satisfied by preservation of the mass in the single finite element volumes. Instead, the energy equation needs additional iteration to gain current pressure and energy update.

The assumed initial conditions considered the motionless air at standard conditions for temperature and pressure. The essential boundary conditions of a rigid wall type were realized by the vanishing of the normal nodal velocity component, where the symmetry planes or v-shape structures were localized. The blast loading was modelled through a specific boundary condition, where the parameters of the incident shock wave, i.e., pressure and mass flow field, acted on the assumed Euler boundary with the application of the so-called ambient zone (elements). ConWep air blast algorithms $[25,26]$ were used to generate the incident shock wave, where the chosen $\mathrm{HE}$ charge was placed in predetermined locations. A description of the infinite domain was achieved by the nonreflecting boundary condition 
applied on the outer boundary, which resulted in the free outflow state.

The geometry of the models was limited to half of the physical system due to its plane symmetry. The Euler domain boundaries were moved away sufficiently to avoid the impact of the possible boundary effects on the problem being investigated. After the discretization process, each model contained between 150 and 200 thousands of 2D quad elements, which provided a high accuracy of calculation.

The main result of the simulation, i.e., the reaction force history, was acquired by summing up all forces recorded for all boundary nodes, which formed the deflector shape. The proper direction (along the symmetry plane) was then selected to further study this value as it evolves with time or its time integral, i.e., impulse. Additionally, colour pressure maps were prepared with the nodal averaging technique to improve readability and interpretation.

The equivalent numerical model was successfully validated by a number of authors in the literature. For example, Schwer in [27] used a very similar model, but in the axis-symmetric domain, studying air blast reflection ratios and angles of incidence. He proved good correspondence between the LS-DYNA results and other experimental and quasianalytical predictions. In turn, Van Dorsselaer et al. [28] performed advanced numerical experimental analysis of shock wave propagation around a convex structure. They concluded that the LS-DYNA Solver delivered pressure results within an experimental uncertainty of $20 \%$. A similar numerical model was investigated by Powell et al. in [29], investigating the aspect of the mesh density dependence. Total impulse characteristics were used as a measure of the results in comparison with different size meshes and convergence analysis with respect to the discretization level.

\section{Results and Discussion}

4.1. Analysis of Deflectors with Multiple V-Shapes. In this section, deflectors with different number of v-shape components are compared. They were situated in such a way that the standoff distance (counted from their base) was equal to $2.6 \mathrm{~m}$ and was fixed for all variants. The assumed distance allowed a sufficiently flat wave front (below 1\%) to be formed, whose flatness was measured according to the following definition:

$$
f=\frac{l-s}{s},
$$

where $l$ is the arc length and $s$ is the chord length for the same central angle. In such an approach, the v-tip positions varied with respect to $\mathrm{HE}$ charge location. When they were closer to the charge, the higher intensity blast wave attacked the protective structures earlier. The deflector location assumption refers to the fact that the vehicle chassis is at a fixed distance from the ground.

The colour map in Figure 1 presents pressure distribution in $2.9 \mathrm{~ms}$ from the charge detonation moment for flat (a), single (b), double (c), and triple (d) deflectors. A specific time was chosen for the analysis because it is at this moment that a reflected wave front is formed for a flat surface. The phenomenon was different for each structure: normal reflection was observed in the case of Vf800, while in all other cases, oblique reflections were registered. In the last two variants, multiple reflections were identified. Surprisingly, in the case of Vf800, the pressure level was not the highest though it covered the whole area of the flat deflector. The lowest value $0.5 \mathrm{MPa}$ was registered on the reflected shock wave front in the case of Vs800, which means that relatively small amount of energy was transferred to the vehicle body. In contrast, the highest pressure was identified for both double and triple deflectors and was localized in the limited space where the adjacent triangles meet. This effect may be considered to be an advantage because of the local action of the pressure but may also have a negative impact if a deflector is perforated due to insufficient strength of material.

An interesting matter is the interpretation and analysis of wave phenomena associated with the interaction of the incident wave with geometrically complex shapes. The first part of the process depicted in Figure 2 was separate reflections from each segment, which created primary wave fronts heading towards each other. It should be noted that the pressure distribution symmetry was violated in reference to a single triangular shape because of the presence of adjacent objects. The waves' collision formed a secondary reflected wave (Figure 2(c)) in the area around the symmetry axis. The wave propagated towards the point of detonation with decreasing intensity because of its divergent character. A rapid fall of pressure in points denoted by $\mathrm{R}$ was the result of the rarefaction effect, which may have an impact on measured parameters. The interference of incident and primary reflected waves is illustrated as the area denoted by letter I but is not expected to influence the issue being studied.

The graph presented in Figure 3 illustrates the reaction force history for all previously mentioned deflector types. The reference plate Vf800 generated the highest reaction force, while a single deflector Vs800 presented the best properties-in this case, it was more than twice smaller. An increased number of individual structures placed instead of a single deflector (Vd800, Vt800, and Vq800) caused a steeper reaction force curve with a higher peak. A simulation of a quadruple deflector showed results close to the reference flat panel. Nevertheless, shaped panels provide some protection against blast with increasing effectiveness in inverse proportion to the number of v-shapes. Furthermore, starting from $3.3 \mathrm{~ms}$ for multishape variants, the time force history did not differ significantly. The results achieved correspond well to the fact that deflectors which consist of numerous $\mathrm{v}$-shapes do not have space scale comparable to the reflection phenomenon [12].

Information about maximum force and momentum transfer is presented in Table 2. The study of momentum transfer showed that the difference between Vf800 and Vs800 was not so drastic because the single deflector was subjected to a stronger wave and load, which lasted for a longer period of time. The analogical parameters for others 


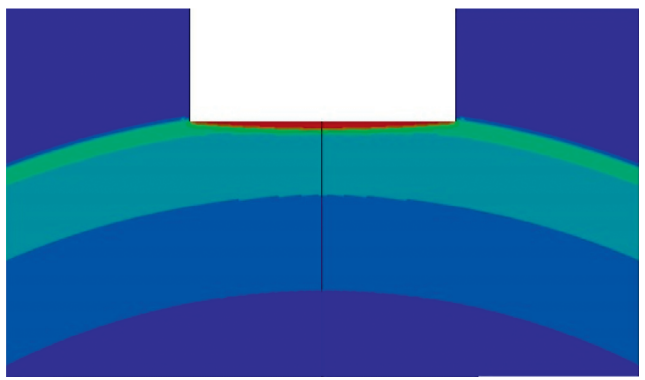

(a)

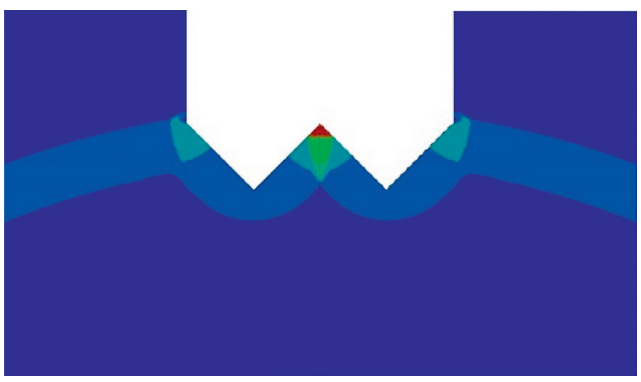

(c)

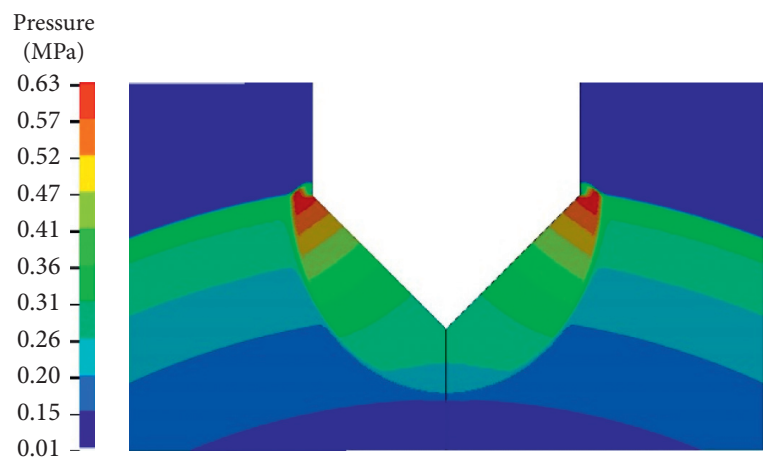

Pressure

(MPa)

0.48

0.41

$0.37-$

$0.33-$

0.29

0.25

0.21

0.18

0.14

(b)

$\begin{gathered}\text { Pressure } \\ (\mathrm{MPa})\end{gathered}$
1.4
1.3
1.1
1.0
0.88
0.75
$0.62-$
0.49
0.36
0.23
0.10
0.10

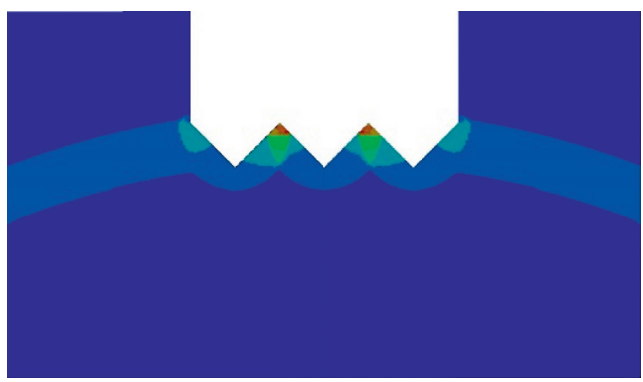

Pressure

(MPa)

$1.4-$
$1.3-$
$1.1-$
$1.0-$
$0.88-$
$0.75-$
$0.62-$
$0.49-$
$0.36-$
$0.23-$
$0.10-$

(d)

Figure 1: Pressure map in $2.9 \mathrm{~ms}$ for different types of deflectors: (a) Vf800, (b) Vs800, (c) Vd800, and (d) Vt800.

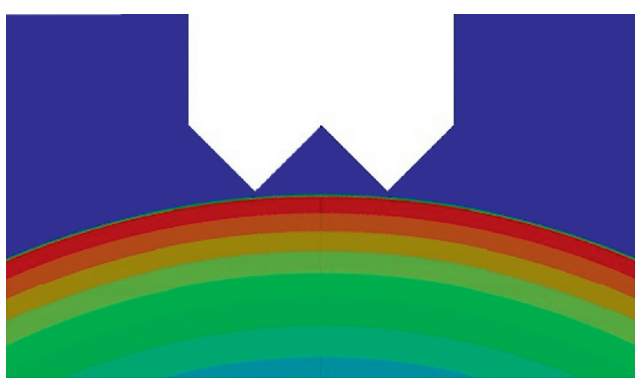

(a)

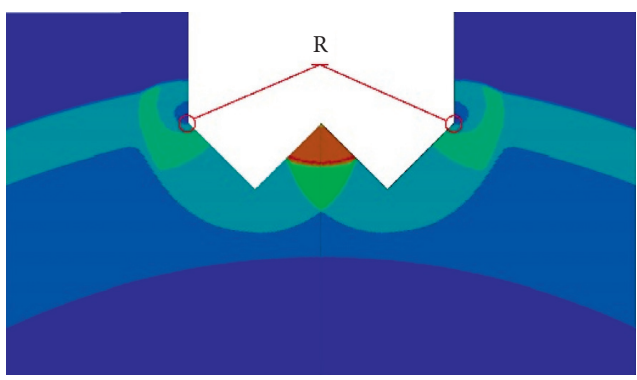

(c)
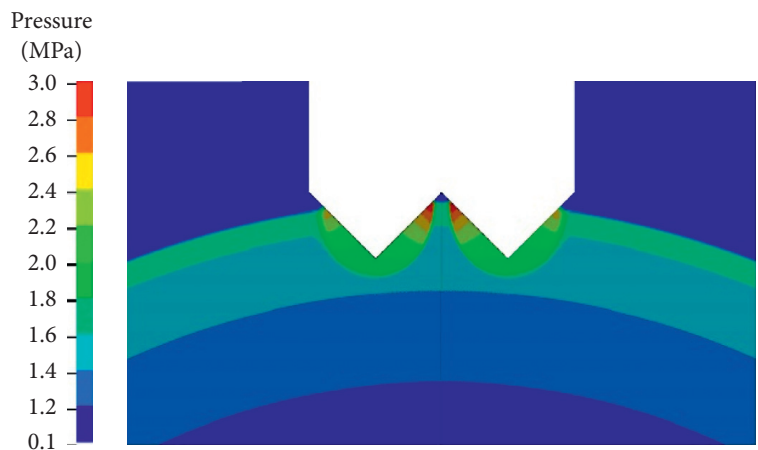

(b)

$$
\begin{gathered}
\begin{array}{c}
\text { Pressure } \\
(\mathrm{MPa})
\end{array} \\
8.1 \\
7.4 \\
6.6 \\
5.9 \\
5.2 \\
4.4 \\
3.7 \\
3.0 \\
2.2 \\
1.5 \\
0.08
\end{gathered}
$$

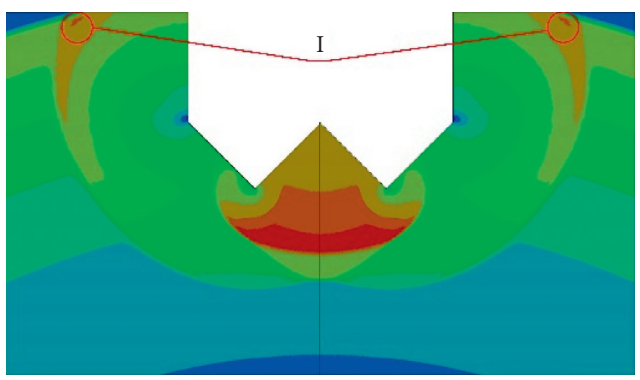

Pressure $(\mathrm{MPa})$

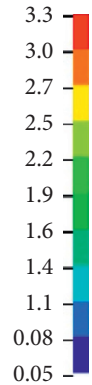

(d)

Figure 2: Process of shock wave reflection in the case of double deflector: (a) $2.4 \mathrm{~ms}$, (b) $2.8 \mathrm{~ms}$, (c) $3.1 \mathrm{~ms}$, and (d) $3.6 \mathrm{~ms}$. 


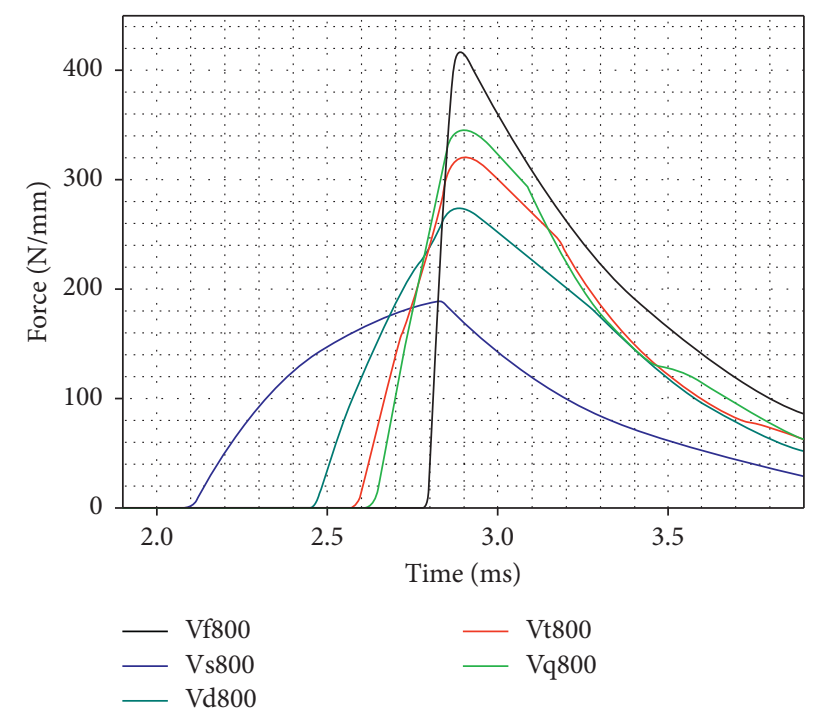

Figure 3: Reaction force history for flat, single, and multiple v-shape deflectors.

TABle 2: Maximum force and momentum transfer.

\begin{tabular}{lcccc}
\hline Variant & \multicolumn{2}{c}{ Maximum force } & \multicolumn{2}{c}{ Momentum transfer } \\
& $(\mathrm{N} / \mathrm{mm})$ & $(\%)$ & $(\mathrm{Nmm} / \mathrm{mm})$ & 272 \\
\hline Vf800 & 416 & 100 & 194 & 71 \\
Vs800 & 189 & 45 & 239 & 88 \\
Vd800 & 274 & 66 & 247 & 91 \\
Vt800 & 320 & 77 & 251 & 92 \\
Vq800 & 345 & 83 & & \\
\hline
\end{tabular}

ranged from $88 \%$ to $92 \%$ of the reference variant. The results for the assumed scenario investigated in this paragraph proved that it is difficult to isolate a pure geometrical effect, and therefore the following research was conducted.

4.2. Analysis of Single Deflector with Different Sizes. This section focuses on the study of the size aspect of single deflectors, whose dimensions varied from 800 to $80 \mathrm{~mm}$. The standoff distance was the same as in Section 4.1. Due to the variable width of deflectors, a normalization procedure was essential for the comparison of quantitative parameters. The authors suggested the following formula:

$$
F_{c}=F_{r} \frac{w_{r}}{w_{a}}
$$

where $F_{c}$ is the calculated force, $F_{r}$ is the registered force, $w_{r}$ is the reference width, and $w_{a}$ is the actual width.

The results shown in Figure 3 were a consequence of two effects: (1) a different deflection process due to the size reduction of $\mathrm{v}$-shape objects and (2) modification of air flow caused by neighbouring structures. It is important from a scientific point of view to separate these phenomena in the analysis process. Therefore, in this section, only the size of a single deflector is investigated, regardless of its applicability. The mechanics behind energy and momentum transfer is the same as the single deflector already discussed, so pressure distributions were not included in this section. However, the reaction forces presented in Figure 4 showed that minimizing the $\mathrm{v}$-shape deflector had a negative impact on its blast mitigation effectiveness for both assumed scenarios, i.e., fixed position of the base (a) and the tip (b) of the deflector according to HE charge location. Although, even for a small Vs80 structure, the maximum reaction force was reduced by a third in reference to Vf800, and it was not as effective as the single deflector Vs800, which was loaded by a significantly stronger wave. Increasing the deflector size led to a reduction of response force in a monotonic manner. In addition, starting from $3.9 \mathrm{~ms}$, the force history was indistinguishable from any of the $\mathrm{v}$-shape sizes studied. The results depicted in Figure 4(b) confirm the superiority of the single large deflector where the monotonic character of force decay is preserved as above. A smaller standoff distance resulted in a much higher value of force peak for all cases because of the more intensive wave.

4.3. Analysis of Boundary Effects. All the variants examined showed that the application of a smaller deflector resulted in deterioration of the blast mitigation ability. However, the issue of the rarefaction effect had not yet been investigated. Two structures with the same position and width were therefore subjected to a blast load. In the first one, adjacent objects were present (Vq800), while in the other, there was only a single $\mathrm{v}$-shape (Vs200). The results obtained are 


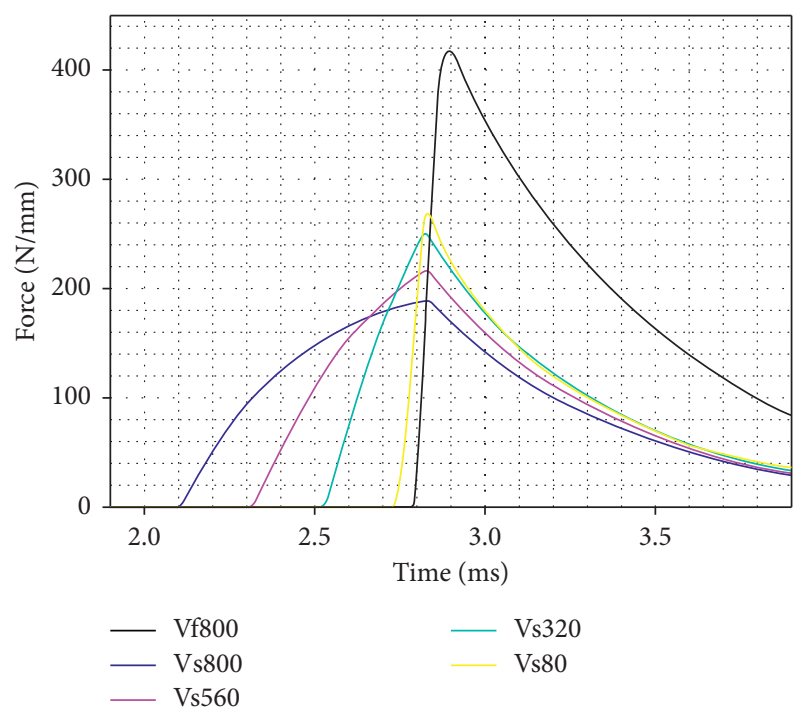

(a)

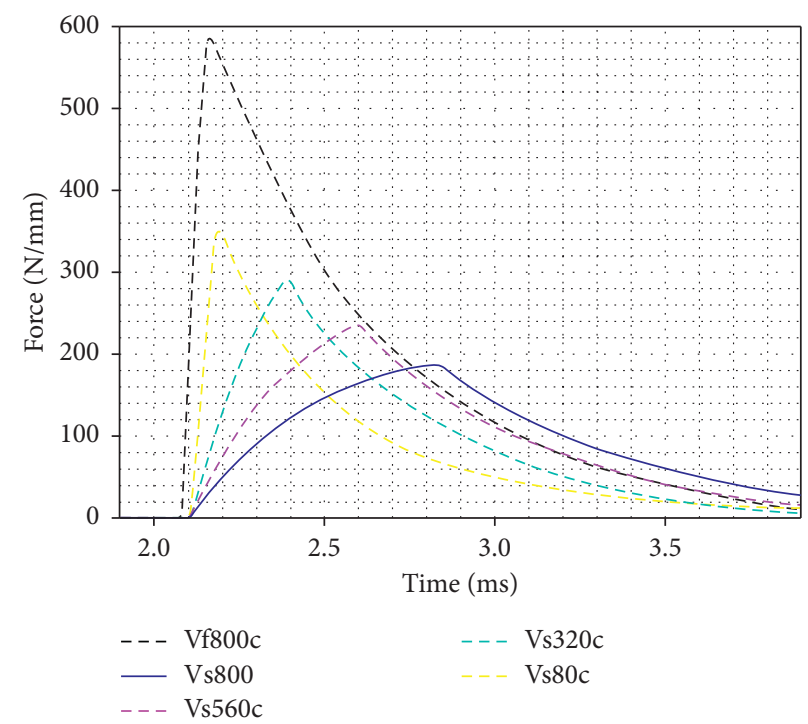

(b)

Figure 4: Reaction force for triangle deflectors: (a) position of the bottom is fixed and (b) position of the top is fixed.

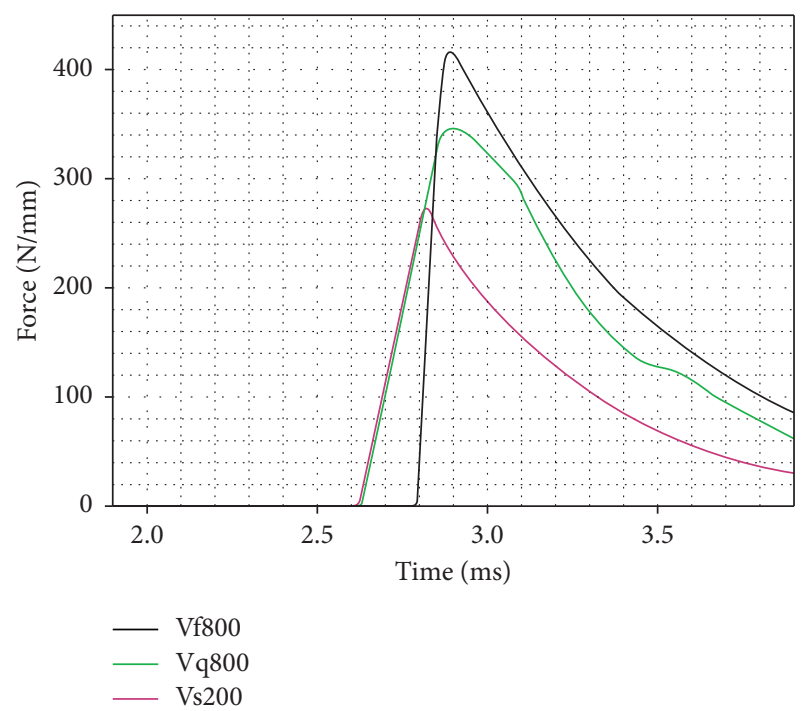

Figure 5: Reaction force for flat, quadruple, and single deflectors.

depicted in Figure 5 with the normalization procedure applied. The force history observed for Vq800 was a superposition of two contrary events: (1) existing rarefaction effect and (2) appearance of secondary reflecting waves. The fact that the variant produced a similar response to the reference case indicated that the first phenomena was not a crucial factor, while the second one might play an important role. In Vs200, rarefaction was not accompanied by other important effects, apart from the primary oblique reflection. The force history (Figure 5) shows deterioration of the efficacy of the multiple v-shape deflector.

\section{Conclusions}

The paper deals with a $2 \mathrm{D}$ numerical study of the blast mitigation ability of multi-v-shape deflectors. Three sets of analysis were conducted: (1) multiplication of triangle components, (2) influence of deflector size, and (3) geometry-induced shock dynamics. The primary measure of the results was reaction force history though momentum transfer and pressure distribution maps were also taken into consideration. The conclusions obtained for plain problem formulation can only be used qualitatively in real conditions though a high accuracy of results is guaranteed due to highdense discretization of space.

The investigation proved that a single deflector achieves a much weaker reaction response. Furthermore, the force increased much more slowly in comparison with the reference flat plate, even though Vs800 was subjected to a stronger wave and load lasted for a longer period of time, which was confirmed by poorer impulse characteristics. The main mechanism for blast mitigation was oblique reflection and rarefaction effects. However, the existence of multiple v-shapes was responsible for a negative effect in the form of secondary reflection waves. Obviously, the impact of the standoff distance was crucial; therefore, a few scenarios were proposed to separately analyse the various mechanical and geometric effects. Studies showed that decreasing the size of the single deflector caused the monotonic effectiveness to deteriorate in both assumed scenarios, i.e., fixed position of its base and tip with reference to the HE charge location. Analysis of adjacent $\mathrm{v}$-shapes' presence showed that their interaction led to increase in the force peak in comparison with a single component (Vs200), where a rarefaction effect was dominant even though the normalization procedure was utilized.

In summary, the results obtained showed that both decreasing the v-shape deflector size and surrounding it by neighbouring structures had a negative influence on its blast mitigation effectiveness. However, for each multishape deflector, some improvement in the protection level was 
present. For situations where installation of a typical protector is not possible, mainly due to dimensional requirements, it may offer a compromise solution.

\section{Data Availability}

The input data (key file) for the LS-DYNA code used to support the findings of this study are available from the corresponding author upon request.

\section{Conflicts of Interest}

The authors declare that they have no conflicts of interest.

\section{Acknowledgments}

This work was supported by the National Centre for Research and Development, Poland (Grant no. DOBR-BIO4/ 022/13149/2013).

\section{References}

[1] Mines Action Canada, Landmine Monitor Report 2009, Mines Action Canada, Ottawa, Canada, 2009.

[2] NATO, Procedures for Evaluating the Protection Level of Armoured Vehicles-Mine Threat, Vol. 2, NATO, Brussels, Belgium, 2014.

[3] A. Shukla and D. S. Rajapakse, Blast Mitigation-Experimental and Numerical Studies, Springer, Berlin, Germany, 2010.

[4] N. Uddin, Blast Protection of Civil Infrastructures and Vehicles Using Composites, Woodhead Publishing, Sawston, UK, 2010.

[5] G. N. Nurick and G. C. Shave, "The deformation and tearing of thin square plates subjected to impulsive loads-An experimental study," International Journal of Impact Engineering, vol. 18, no. 1, pp. 99-116, 1996.

[6] A. C. Jacinto, R. D. Ambrosini, and R. F. Danesi, "Experimental and computational analysis of plates under air blast loading," International Journal of Impact Engineering, vol. 25, no. 10, pp. 927-947, 2001.

[7] S. Ambrosini and G. Slawinski, "The influence of utilizing different materials and their configurations on ballistic panels blast resistance," in Advances in Mechanics: Theoretical, Computational and Interdisciplinary Issues, CRC Press, Boca Raton, FL, USA, 2016.

[8] I. S. Sandhu, "Mitigation of blast induced acceleration using open cell natural rubber and synthetic foam," Defence Science Journal, vol. 69, no. 1, pp. 53-57, 2019.

[9] C. Qi, "Dynamic response and optimal design of curved metallic sandwich panels under blast loading," The Scientific World Journal, vol. 2014, Article ID 853681, 14 pages, 2014.

[10] W. Cheng, "Numerical analysis of cladding sandwich panels with tubular cores subjected to uniform blast load," International Journal of Impact Engineering, vol. 133, 2019.

[11] S. Guangyong, "Dynamic response of sandwich panel with hierarchical honeycomb cores subject to blast loading," ThinWalled Structures, vol. 142, pp. 499-515, 2019.

[12] G. B. Dor, Shock Wave Reflection Phenomena, SpringerVerlag, Berlin, Germany, 1992.

[13] V. Denefeld, N. Heider, A. Holzwarth, A. Sättler, and M. Salk, "Reduction of global effects on vehicles after IED detonations," Defence Technology, vol. 10, no. 2, pp. 219-225, 2014.
[14] J. Trajkovski, J. Perenda, and R. Kunc, "Blast response of Light armoured vehicles (LAVs) with flat and V-hull floor," ThinWalled Structures, vol. 131, pp. 238-244, 2018.

[15] R. Gieleta, W. Barnat, and T. Niezgoda, "Experimental investigation of deflector's angle influence on energy absorption," Journal of KONES. Powertrain and Transport, vol. 19, no. 4, pp. 201-205, 2015.

[16] S. Barnat, G. S. Langdon, G. N. Nurick, E. G. Pickering, and V. H. Balden, "Response of V-shape plates to localised blast load: experiments and numerical simulation," International Journal of Impact Engineering, vol. 46, pp. 97-109, 2012.

[17] P. Kumar, J. LeBlanc, and A. Shukla, "Effect of curvature on shock loading response of aluminum panels," Dynamic Behavior of Materials, Volume 1, vol. 1, pp. 369-374, 2011.

[18] J. LeBlanc, R. Kunc, and I. Prebil, "Blast response of centrally and eccentrically loaded flat-, U-, and V-shaped armored plates: comparative study," Shock Waves, vol. 27, no. 4, pp. 583-591, 2017.

[19] G. Gurumurthy, Blast Mitigation Strategies for Vehicles Using Shape Optimization Methods, Massachusetts Institute of Technology, Cambridge, MA, USA, 2008.

[20] C. Davis, Prevention of Injury in Mine Resistant Ambush Protected (MRAP) Vehicle Accidents, United States Army Aeromedical Research Laboratory, Fort Rucker, AL, USA, 2013.

[21] R. Hajek, M. Foglar, and J. Fladr, "Influence of barrier material and barrier shape on blast wave mitigation," Construction and Building Materials, vol. 120, pp. 54-64, 2016.

[22] K. Genson, "Vehicle shaping for mine blast damage reduction," University of Maryland, College Park, MA, USA, Master's of Science, 2006.

[23] A. Morka, "Numerical analysis of momentum transfer in the case of blast protection structures," AIP Conference Proceedings, vol. 2078, no. 1, 2019.

[24] J. Hallquist, LS-Dyna Theory Manual, LSTC, Livermore, CA, USA, 2018.

[25] G. Randers-Pehrson and K. Bannister, AirBlast Loading Model for DYNA2D and DYNA3D, Army Research Laboratory, Adelphi, MD, USA, 1997.

[26] C. N. Kingery and G. Bulmash, Air-Blast Parameters from TNT Spherical Airburst and Hemispherical Surface Burst, U.S. Army Ballistic Research Laboratory, Aberdeen Proving Ground, MD, USA, 1984.

[27] L. Schwer, "Air blast reflection ratios and angle of incidence," in Proceedings of the 11th European LS-DYNA Conference, Salzburg, Austria, 2017.

[28] N. Van Dorsselaer, S. Eveillard, and S. Trelat, "Experiments and simulations of explosives: shock wave propagation around a convex structure," in Proceedings of the 15th International LS-DYNA Users Conference, Detroit, MI, USA, June 2018.

[29] D. A. Powell, D. Bogosian, and L. Schwer, "Mesh sensitivity of blast wave propagation," in Proceedings of the 15th International LS-DYNA Users Conference, Detroit, MI, USA, June 2018. 